Nervenarzt 2007 · 78:1303-1309

DOI 10.1007/s00115-007-2309-x

Online publiziert: 6. Juli 2007

๑) Springer Medizin Verlag 2007
B. Waldvogel ${ }^{1} \cdot$ A. Ullrich ${ }^{2} \cdot$ H. Strasburger ${ }^{3,4}$

${ }^{1}$ Praxis für Psychotherapie, München

2 Praxiszentrum Neurologie Psychiatrie München-Mitte, München

${ }^{3}$ Abteilung für Medizinische Psychologie der Universität Göttingen

${ }^{4}$ Institut für Medizinische Psychologie der Ludwig-Maximilians-Universität München

\title{
Blind und sehend in einer Person
}

\section{Schlussfolgerungen zur Psychoneurobiologie des Sehens}

\section{Fallbericht}

\section{Anamnese}

Aus einer psychiatrischen Klinik wurde dem Erstautor eine damals 33-jährige blinde Patientin mit der Diagnose einer multiplen Persönlichkeit(sstörung) [ICD 10: F44.81] zur psychotherapeutischen Behandlung überwiesen. Sie erschien mit ihrem Blindenführhund und berichtete hinsichtlich ihrer Blindheit, 13 Jahre zuvor ein unfallbedingtes Schädel-Hirn-Trauma erlitten zu haben. Dieses habe zunächst eine erhebliche Sehbehinderung bedingt, die dann allmählich zu einer vollständigen Blindheit vorangeschritten sei. In einer der folgenden Sitzungen legte sie auf Bitten des Erstautors ein wissenschaftliches Gutachten einer Universitätsaugenklinik über ihre Blindheit vor, das nach ihrer vollständigen Erblindung von einem Sozialhilfeträger in Auftrag gegeben worden war.

Das Gutachten sollte die Fragestellung beantworten, „ob die subjektiven Angaben der Patientin dem objektiven Befund entsprechen“. Diese Frage wurde auf der Basis der Diagnose einer kortikalen Blindheit (Rindenblindheit) bejaht. Die Untersuchung des vorderen Augenabschnitts mittels Spaltlampe, des Kammerwinkels (Gonioskopie), des Augenhintergrundes (Dreispiegelkontaktglas) sowie des Augeninnendrucks (Applanationstonometer) ergaben keinerlei Hinweise auf eine organische Beeinträchtigung des Auges. Die objektive Refraktometrie (Humphrey) ergab eine leichte Myopie (2 Dpt), die objektive Visusbestimmung mit der Nystagmustrommel (Kotowsky) einen herabgesetzten aber nachweisbaren Visus (OS und $O D=0,1)$. Der gleiche Befund der Intaktheit des peripheren Apparats ergab sich bezüglich der Augenmotorik: korrekte Parallelstellung, intakte Augapfelbeweglichkeit (d. h. intakte Kommandobewegungen). Dagegen fehlten jegliche Augenfolgebewegungen. Ebenso zeigten alle Tests der subjektiven Sehfunktion einen fast vollständigen Sehverlust: Laserinterferenz-Visusbestimmung, Kugelperimetrie am Goldmann-Perimeter und Stereopsistests (Worth und Bagolini) nicht durchführbar. Bemerkenswert ist insbesondere das Ausbleiben reflektorischer Schutzreaktionen auf grelles Licht (kein Tränen, Kneifen, keine Schreckreaktion). Eine vorhandene Restfunktion bestand darin, dass das Vorhandensein eines grellen Lichts nah am Auge detektiert wurde, mit Farbempfindung nach Abschalten des Lichts (durch Netzhautnachbilder erklärbar), wobei die Lichteinfallsrichtung nicht angegeben werden konnte.

Entscheidend aber ist die Bestätigung des Befunds bezüglich der subjektiven Funktion mittels musterevozierter Po- tenziale (durchgeführt an einer Universitätsaugenklinik). Trotz guter Mitarbeit (Infrarotfixationskontrolle) waren weder bei foveal ( $3^{\circ}$ Sehwinkel), noch perifoveal $\left(13^{\circ}\right)$, noch großflächig $\left(30^{\circ}\right)$ dargebotenen (Schachbrett-)Musterwechselreizen evozierte Potenziale nachweisbar. Demgegenüber zeigten blitzevozierte Potenziale normale Antworten, mit normaler Amplitude und normaler Latenz der P10o. Dies belegt das Fehlen adäquater Aktivität der Sehrinde bei dargebotenen Musterreizen und bestätigt das Ausbleiben (subjektiver) strukturierter Wahrnehmung, bei verbliebener Fähigkeit der Lichtdetektion.

\section{Diagnostik}

Die Überweisungsdiagnose einer multiplen Persönlichkeit(sstörung) bestätigte sich in der aufgenommenen psychotherapeutischen Behandlung recht bald durch spontane „Identitätswechsel“ der Patientin. Es präsentierten sich nach und nach über 10 verschiedene Persönlichkeitsanteile, die sich durch je eigene $\mathrm{Na}$ men, verschiedene Altersangaben, unterschiedliche Geschlechtsidentitäten, unterschiedliche Einstellungen, Neigungen, Temperamentsunterschiede und weitere charakterliche Merkmale auszeichneten und identifizieren ließen. Auch Stimme, Gestik und Mimik waren jeweils deutlich unterscheidbar. In einigen dieser Persön- 
lichkeitszustände konnte sich die Patientin nur in Englisch verständigen, in anderen nur in Deutsch, in einigen war sie beider Sprachen mächtig. Sie hatte einige Jahre ihrer Kindheit und Jugend in einem englischsprachigen Land verbracht und dort nur Englisch gesprochen.

Aufgrund dieser Beobachtungen, die auch mit fremdanamnestischen Angaben durch Freunde der Patientin und mit den Beobachtungen in der psychiatrischen Klinik, in der sie unmittelbar vor Beginn der Psychotherapie behandelt wurde, übereinstimmten, waren alle Kriterien des DSM-IV für die Diagnose einer dissoziativen Identitätsstörung erfüllt. Die Bezeichnung „dissoziative Identitätsstörung “ ist gegenüber dem noch in der ICD-10 verwendeten Begriff „multiple Persönlichkeit(sstörung)“ definitorisch treffender und hat sich in der einschlägigen Literatur auch seit vielen Jahren durchgesetzt [3]. Für eine operationalisierte Diagnostik hat sich unter mehreren Screeninginstrumenten und Interviewverfahren das Structured Clinical Interview for DSM-IV Dissociative Disorders SCID-D [20] als besonders reliables und valides Diagnoseinstrument bewährt [21], das als Strukturiertes Klinisches Interview für Dissoziative Störungen SKID$\mathrm{D}$ auch in deutscher Übersetzung vorliegt [4]. Auch nach dieser Operationalisierung der DSM-IV-Kriterien durch das SCID-D war bei der Patientin die Diagnose einer dissoziativen Identitätsstörung zu stellen.

\section{Therapie und Verlauf}

Im 4. Jahr der psychotherapeutischen Behandlung ${ }^{1}$, zu der die Patientin stets mit ihrem Blindenführhund erschien, konnte die Patientin unmittelbar nach einer Behandlungsstunde in einem ihrer jugendlichen männlichen Identitätszustände plötzlich einzelne Worte auf der Titelseite einer Zeitschrift erkennen. Diese Wahrnehmungsfähigkeit beschränkte sich zunächst nur auf ganze Wörter (die das Wort bildenden Buchstaben konnten nicht einzeln erkannt werden) und auf diesen einen Persönlichkeitsanteil. In den folgenden Behandlungsstunden konnte diese Fähig-

1 Zur Psychotherapie dissoziativer Identitätsstörungen $\mathrm{s} .[2,16]$. keit zunächst auf besonders hell beleuchtete Objekte und dann relativ rasch auf die gesamte visuelle Wahrnehmungswelt generalisiert werden. Mittels hypnotherapeutischer Techniken konnte dieser Effekt sodann auch auf andere Persönlichkeitsanteile generalisiert werden.

Über einige Monate hinweg existierten nun gleichzeitig zunehmend mehr Persönlichkeitsanteile, die vollständig sehen konnten, und zunehmend weniger Persönlichkeitsanteile, die weiterhin vollständig blind waren, wobei diese verschiedenen Zustände der Sehfähigkeit sekundenschnell alternieren konnten.

\section{Visuell evozierte Potenziale im sehenden und im blinden Zustand}

In dieser Phase der gleichzeitigen Existenz sehender und blinder Persönlichkeitszustände wurde eine Ableitung visuell evozierter Potenziale vorgenommen, um einerseits eine unabhängige Überprüfung der früher gewonnenen Resultate zu erlangen und andererseits zu prüfen, inwieweit die Angaben der Patientin mit physiologischen Korrelaten einhergehen.

\section{Methoden}

Bei den Messungen kamen (wie auch zuvor) die in der neuroophthalmologischen Praxis zur Befundung der Intaktheit des primären Übertragungsweges gängigen Schachbrettmusterumkehr-VEP zum Einsatz (vgl. etwa [9]). Die Reizdarbietung geschah an einem 12-Zoll SchwarzWeiß-Monitor (JVC TM-122), angesteuert durch ein VEP/EMG-Erfassungssystem (Neuropack 2, Hersteller Nihon Kohden). Die Reizung erfolgte binokular mit Visuskorrektur. Bei dem verwendeten Betrachterabstand von $150 \mathrm{~cm}$ ergab sich eine Reizfläche von $6,7^{\circ} \times 9,3^{\circ}$ Sehwinkel $(17,5 \mathrm{~cm} \times 24,3 \mathrm{~cm})$. Es wurden niedrige und hohe Ortsfrequenzen (große bzw. kleine „Karos“) eingesetzt ( $6 \times 8$ bzw. $45 \times 60$ Musterelemente), mit einer Elementgröße von $1,2^{\circ}$ bzw. $0,15^{\circ}$ Sehwinkel. Die Modulationsfrequenz betrug $1 \mathrm{~Hz}$ und $10 \mathrm{~Hz}$, d.h. es wurden sowohl transiente wie stationäre („Steady-state-)Potenziale“ erfasst. Die Signalerfassung (Vorund Hauptverstärker, Signalanalyse) geschah an der gleichen Anlage (Bandpass 0,5-100 Hz, 50-Hz-Sperrfilter, 32 Mitte- lungen). Die differente Elektrode wurde entsprechend $\mathrm{Oz}$, die indifferente entsprechend Fpz platziert (entsprechend internationalem 10-20-System). Die Elektrodenimpedanzen lagen unter $5 \mathrm{kOhm}$.

Die VEP-Ableitungen dauerten jeweils nicht länger als 2 min, es wurden Pausen von 5 min eingelegt. Die Reihenfolge der aktivierten Persönlichkeitszustände wurde teilbalanciert (Reihenfolge: sehend, blind, sehend), um auszuschließen, dass durch Ermüdung (trotz der Ruhepausen) Amplitudenabnahmen oder Fixationsunregelmäßigkeiten auftraten. Die VEP-Latenzen des transienten VEP sind gegenüber Ermüdung ohnehin weitgehend indifferent.

Ein zusätzlicher Beobachter kontrollierte die Augenstellung der Patientin während der gesamten Messdauer. Somit wurde sichergestellt, dass auch im blinden Zustand ( $\mathrm{S}-$ ) unveränderte Vigilanz vorlag, die Augen geöffnet blieben, keine verstärkte Blinzelreaktion auftrat und gleicher Fixationsort (innerhalb etwa $2^{\circ}$ ) vorlag. Ausgeschlossen wurde hierdurch insbesondere auch Schielen oder Fixieren von Randbereichen des Monitors oder Punkten ganz außerhalb.

Die Untersuchung wurde in einem tageslichtlosen Raum mit abgedimmter, gleichbleibender Beleuchtung durchgeführt; sie geschah gegen 18 Uhr abends. Zum Zeitpunkt der Untersuchung war die Probandin wach, positiv motiviert und entspannt. Die Untersuchungen wurden in Begleitung des Therapeuten durchgeführt.

\section{Ergebnisse}

Zunächst wurde die Probandin im Zustand der Normalsichtigkeit, d. h. mit den visuskompetenten Persönlichkeitsanteilen (S+) untersucht. Es fand sich bei niedriger und hoher Ortsfrequenz und bei Stimulationsfrequenzen von $1 \mathrm{~Hz}$ wie auch $10 \mathrm{~Hz}$ ein gut reproduzierbares kortikales Antwortpotenzial mit Latenz von 104$106 \mathrm{~ms}$ und Amplitude über $10 \mu \mathrm{V}$. Die Ergebnisse waren jeweils reproduzierbar (- Abb. 1). Dann wurde ein „blinder“ Persönlichkeitszustand durch Aufrufen seines Namens aktiviert. In diesem $\mathrm{Zu}$ stand der fehlenden subjektiven Sehfähigkeit (S-) ließen sich weder bei transienter ( $1 \mathrm{~Hz})$ noch stationärer (10 Hz) Reizung, 
Hier steht eine Anzeige.

算 Springer 


\section{Kasuistiken}

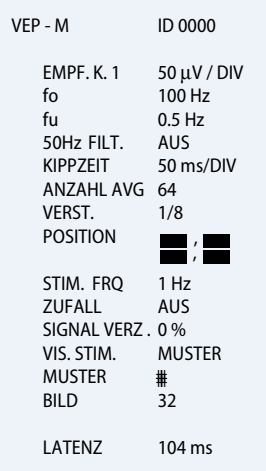

a

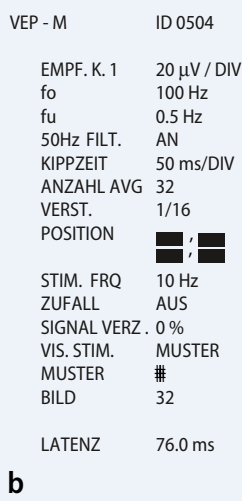

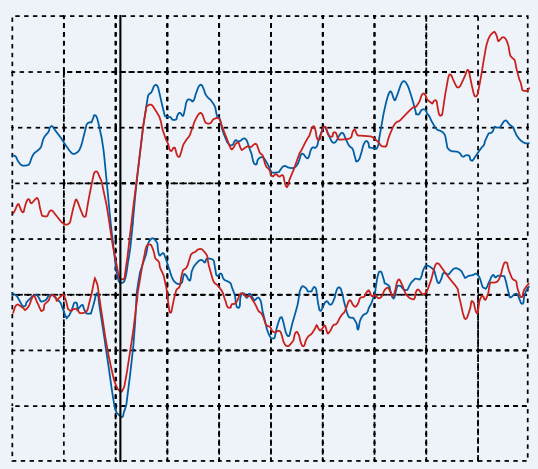

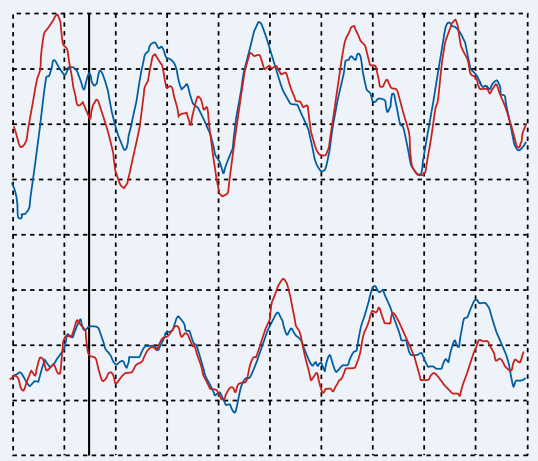

Abb. $1 \Delta$ Musterevozierte Potenziale im sehenden Persönlichkeitszustand, bei a transienter $(1 \mathrm{~Hz})$ und b „Steady-state-“ $(10 \mathrm{~Hz})$ Reizung (die Spuren sind zur besseren Unterscheidung farblich nachgezeichnet)

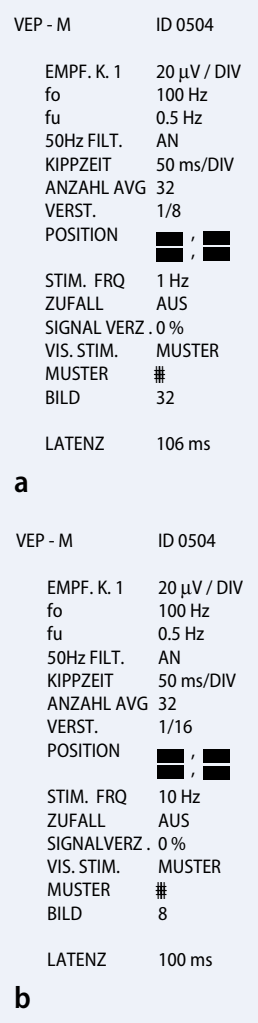

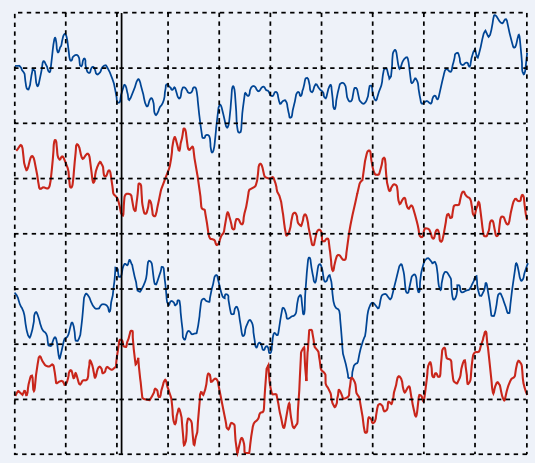

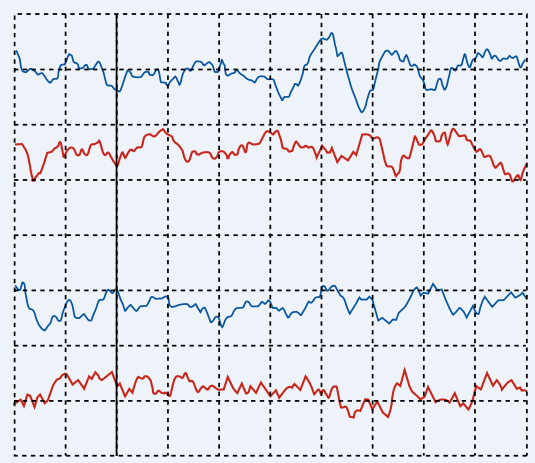

Abb. 2 A Musterevozierte Potenziale im blinden Persönlichkeitszustand, bei a transienter $(1 \mathrm{~Hz})$ und b „Steady-state-“ (10 Hz) Reizung

weder bei niedrigen noch hohen Ortsfrequenzen reproduzierbare VEP-Antworten auslösen (• Abb. 2).

\section{Diskussion}

Psychogen bedingter Verlust (bewusster) visueller Wahrnehmung wurde bereits Ende des 19. Jahrhunderts u. a. von Charcot und von Freud beschrieben. Bemerkenswert an dem vorliegenden Fall ist die während der visuellen Genesung zu beobachtende Koppelung der Sehfähigkeit an Persönlichkeitsanteile einer strukturell dissoziierten Persönlichkeit. Ebenso bemerkenswert sind die - nach jahrelanger vollständiger Erblindung mit allen Anzeichen einer organischen Ursache - plötzlich eintretende partielle Sehfähigkeit und im Weiteren die Wechsel zwischen scheinbar vollständiger Blindheit und nahezu uneingeschränktem Sehvermögen.

Dieser kurzfristig mögliche Wechsel zeigt, dass die einfache Rückbildung einer zentralnervösen organischen Schädigung als Grund der Wiederherstellung der Sehfähigkeit nicht in Frage kommt: Auch wenn Plastizität visueller Hirnfunktion lange nach einer hirnorganischen Schädigung heute nicht mehr grundsätzlich in Frage gestellt wird, so sind doch sowohl Spontanheilung neuronaler Funktionen als auch der Erfolg eines neurorehabilitativen Funktionstrainings [10] langwierige, zähe Prozesse mit jeweils kleinen Fortschritten über die Zeit. Auch tritt Spontanheilung zerebraler Sehschäden in aller Regel im zeitlichen Anschluss an die Schädigung auf (Trainingserfolge scheinen hingegen von der Zeit nach Läsion unabhängig [15]). Eine Reorganisation synaptischer Verbindungen nach SHT erscheint damit als Basis der Wiedergewinnung der Sehfunktion im vorliegenden Fall ausgeschlossen. Wir vermuten vielmehr, dass das SHT und eine dadurch möglicherweise bedingte vorübergehende Beeinträchtigung des Sehens eine Bahnung für eine „psychogene" Erblindung bildete.

Das Phänomen einer temporären und jederzeit reversiblen Unterdrückung des gesamten neuronalen visuellen Signals zumindest eines Auges ist weit weniger ungewöhnlich als dies vielleicht den Anschein hat: Kurzfristig sich widersprechende Informationen der beiden Augen - durch
Schielen oder in der Situation dichoptischer Reizpräsentation (d. h. Darbietung unterschiedlicher Bilder auf dem linken und rechten Auge, etwa mittels Prismen) führen zu temporärem „Ausblenden“ der Sehinformation eines der beiden Augen; schon im Fall ausgeprägter Augendominanz geschieht dies in abgeschwächter Form. Es stehen also durchaus Hirnmechanismen zur Verfügung, die eine weitgehende Modulation der eingehenden Sehinformation über das ganze Gesichtsfeld oder Teile davon gestatten. Auf welcher Ebene dies im Fall dichoptischer Präsentation geschieht ist Gegenstand aktueller Forschung; wahrscheinlichste Stationen sind das Corpus geniculatum laterale (CGL) des Thalamus (oft als „Tor zum Kortex" bezeichnet) und der primäre visuelle Kortex, da alle bewusst verarbeiteten Sehinformationen über diesen primären Pfad eingehen. Entsprechende Belege mittels fMRT liegen nun in der Tat vor [26]. Analog finden Vuilleumier et al. [25] bei „psychogenem“ Verlust von sensomotorischer Funktion neben anderem eine herabgesetzte Aktivität im kontralateralen Thalamus. 
Ein weiterer Mechanismus der temporären, so genannten „Top-down-Modulation" der eingehenden Sehinformation ist der der visuellen örtlichen Aufmerksamkeit („spatial attention“). Diese bereits 1871 von von Helmholtz [7] beschriebene Fähigkeit [22] besteht darin, willkürlich oder unwillkürlich Sehinformation innerhalb umschriebener Bereiche des Gesichtsfeldes verstärkt oder abgeschwächt wahrzunehmen [5]. Auch bei dieser Fähigkeit kommen als neuronaler Ort der Modulation frühe kortikale, extrastriäre Areale [8, 12] oder subkortikale wie das CGL in Betracht und auch hier liegen diesbezügliche fMRT-Belege nun vor [13, 11]. O'Connor et al. [13] etwa konnten in ihrer fMRT-Studie zeigen, dass beachtete Reize zu verstärkter CGL-Aktivität, ignorierte Reize dagegen zu herabgesetzter CGL-Aktivität führten.

Das visuell evozierte Potenzial (VEP), sei es in der klinisch gebräuchlichen Form mittels Schachbrettmusterreizen oder mittels den in der Forschung häufiger eingesetzten Sinusgitterreizen, gilt als zuverlässiger Indikator visuell verursachter Aktivierung früher kortikaler Bereiche [9, 23, 24], wobei die prominenteste Komponente $(\mathrm{P} 100)$ vermutlich in Area $V_{2}$ generiert wird [8]. Allerdings müssen bei der VEPErfassung eine Reihe von Störeinflüssen, insbesondere subjektive Faktoren (Kooperation, Vigilanz, exzentrische Fixation, Defokussieren) bedacht werden. Durch sorgfältige Beobachtung des Probanden (insbesondere im blinden Zustand) sind diese erfahrungsgemäß recht gut zu kontrollieren; auch das äußerlich nicht unmittelbar erkennbare Defokussieren ist in der Regel durch sichtbares Schielen begleitet. Zudem wären beim Defokussieren unterschiedliche Ergebnisse zwischen niedriger und hoher Ortsfrequenz der Reize zu erwarten gewesen, was nicht der Fall war. Genaue Fixation war nicht notwendig, da großflächige Reize verwendet wurden. In der in der Anamnese berichteten gutachterlichen Untersuchung der Patientin fand zudem eine Infrarot-Augenfixationskontrolle statt. Insofern kann für das dort erhaltene Ergebnis ausbleibender VEP eine recht hohe Validität beansprucht werden. Es sei zudem angemerkt, dass VEP keine bewusste Reizverarbeitung oder gleichbleibend hohe Aufmerksamkeit erfordern; auch im anästhesierten Zustand

Nervenarzt 2007 · 78:1303-1309 DOI 10.1007/s00115-007-2309-x

(c) Springer Medizin Verlag 2007

\section{B. Waldvogel $\cdot$ A. Ullrich $\cdot H$. Strasburger Blind und sehend in einer Person. Schlussfolgerungen zur Psychoneurobiologie des Sehens}

Zusammenfassung

Es wird der Fall einer Patientin mit dissoziativer Identitätsstörung vorgestellt, die nach 15-jähriger, als kortikal diagnostizierter Blindheit im Laufe einer psychotherapeutischen Behandlung schrittweise wieder zu sehen begann. Zunächst konnten nur einige wenige Persönlichkeitsanteile wieder sehen, während andere weiterhin blind waren.

Dies konnte durch elektrophysiologische Untersuchungen bestätigt werden, in denen die noch blinden Persönlichkeitsanteile ausbleibende, die sehenden Persönlichkeitsanteile hingegen völlig unauffällige, reguläre evozierte Potenziale aufwiesen. Das Umschalten zwischen sehenden und blinden Anteilen konnte übergangslos geschehen. Als neuronale Grundlage der psychogenen Blind- heit kommt eine „Top-down-Modulation“ der Aktivität der primären Sehbahn, auf Ebene des Thalamus oder primären visuellen Kortex, in Betracht. Mittels VEP (visuell evozierte Potenziale) -Untersuchungen kann daher eine psychogene Blindheit nicht von organischen Ursachen einer Unterbrechung der Sehbahn abgegrenzt werden. Zusammenfassend scheint psychogene Blindheit den Zufluss visueller Information auf früher Stufe zu blockieren.

\section{Schlüsselwörter}

Dissoziation · Dissoziative Identitätsstörung · Konversion · Psychogene Blindheit · Visuell evozierte Potenziale · Sehbahn · Thalamus

\section{Sighted and blind in one person. A case report and conclusions on the psychoneurobiology of vision}

\section{Summary}

We present a patient with dissociative identity disorder (DID) who after 15 years of diagnosed cortical blindness gradually regained sight during psychotherapeutic treatment. At first only a few personality states regained vision, whereas others remained blind. This was confirmed by electrophysiological measurement, in which visual evoked potentials (VEP) were absent in the blind personality states but normal and stable in the seeing states. The switch between these states could happen momentarily. As a neural basis of such psychogenic blindness, we assume a top-down modulation of activity in the pri- mary visual pathway, possibly at the level of the thalamus or the primary visual cortex. Therefore VEPs do not allow distinction of psychogenic blindness from organic disruption of the visual pathway. In summary, psychogenic blindness seems to suppress visual information at an early neural stage.

\section{Keywords}

Conversion disorders · Dissociation - Dissociative identity disorder - Psychogenic blindness · Thalamus · Visual evoked potentials · Visual pathway 
können VEP abgeleitet werden [14]. Völlig auszuschließen sind Störeinflüsse aber nicht und unter dieser Einschränkung müssen unsere Schlussfolgerungen gesehen werden.

Gutachterlich sind VEP das Verfahren der Wahl. Spiegel et al. [19] z. B. nutzten das VEP, um bei hypnotisch induzierter Hemmung visueller Wahrnehmung einen herabgesetzten Informationszufluss $\mathrm{zu}$ objektivieren und konnten eine $\mathrm{Ab}$ nahme der Amplitude der frühen (P10o) und späteren $\left(\mathrm{P}_{3} \mathrm{Oo}\right)$ VEP-Komponenten zeigen. Klassische VEP-Untersuchungen bei Fällen psychogener Blindheit, wie die von Halliday [6] oder Altenmüller et al. [1], fanden durchgehend normales Muster-VEP, woraus die Autoren auf die (organische) Intaktheit der primären Sehbahn schließen.

Der im Gegensatz dazu bei unserer Patientin erhaltene Befund fehlender VEPs nach ihrer Erblindung - bei augenscheinlich vollständig intakter peripherer Sehfunktion - legt daher den Schluss nahe, dass das visuelle neuronale Signal der primären Sehbahn bezüglich Mustererkennung weitgehend blockiert wurde. Insbesondere das bei der Patientin beobachtbare spontane Umschalten - innerhalb von Sekunden - zwischen fehlendem und intaktem VEP kann hierbei als Beleg dynamischer, weitgehender Suppression der Mustersehinformation auf Ebene der Sehbahn oder des Kortex, d. h. des CGL oder $\mathrm{V} 1$, gewertet werden.

Ein interessantes Detail während der Genesung war die Tatsache, dass die Sehfähigkeit zunächst ausschließlich auf das Erkennen ganzer Wörter eingegrenzt war, die einzelnen Buchstaben der gelesenen Wörter - auch wenn sie einzeln dargeboten wurden - dagegen nicht erkannt werden konnten. Erkennen von farbig gedruckten Wörtern mit niedrigem Kontrast und Schrift mit invertiertem Kontrast (helle Schrift auf dunklem Grund) war ebenfalls nicht möglich. Zur Untersuchung wurden der Patientin Texte mit großer Schrift vorgelegt. Dieser Zustand war jedoch zu kurzdauernd, um systematisch untersucht werden zu können.

Eine solche Trennung von Sehfunktionen ist mit experimentalpsychologischen Forschungsergebnissen vereinbar. Untersuchungen zum Lesen ergeben immer wieder, dass das Erkennen von Buchstaben und das von Wörtern nicht fest aneinander gebunden sind, d. h. dass das Erkennen der einzelnen Buchstaben eines Wortes weder notwendig noch hinreichend für das Erkennen des Wortes ist. Ersteres ist eine reine Mustererkennungsleistung (beim Makaken wird dies vermutlich im Inferotemporalkortex geleistet). Das Erkennen von Wörtern ist demgegenüber eine Leistung, die bereits sprachliche Aspekte einschließt, und vermutlich eine Verarbeitung im Wernicke-Areal einschließt. Auch die herabgesetzte Leistung bei niedrigem oder ungewohntem Kontrast ist mit einem herabgesetzten Informationszufluss vereinbar.

Es verbleibt die Frage, wie unterschiedliche Persönlichkeitsanteile unterschiedlichen Zugang zu solchen modulatorischen Zentren erhalten können. Es kann hierauf solange keine Antwort geben, solange über die neurobiologischen Grundlagen des Ich-Erlebens und des Bewusstseins so wenig bekannt ist. Gesichert scheint, dass verschiedene Persönlichkeitszustände mit unterschiedlichen neuronalen Aktivierungsmustern einhergehen, die auch extrastriäre visuelle Areale betreffen $[17,18]$. Nimmt man hypothetisch an, dass einheitliches Erleben einer Person (oder im Fall dissoziativer Identitätsstörungen mehrerer „Persönlichkeitsanteile“) durch Rückkopplungskreise über frontale Hirnregionen vermittelt ist, wie dies gegenwärtig in der Aufmerksamkeitsforschung diskutiert wird, so könnten getrennte Rückkopplungskreise separat Zugriff auf früh liegende, modulatorische Zentren besitzen. Das Umschalten zwischen Persönlichkeitsanteilen und zugehörigen sensorischen Modulationen würde auf diese Weise grundsätzlich verstehbar.

\section{Korrespondenzadresse \\ Dr. B. Waldvogel}

Praxis für Psychotherapie

Enhuberstraße 1, 80333 München

mail2@bruno-waldvogel.de

Danksagungen. Wir danken Torsten Wüstenberg (Universität Göttingen) für die drucktechnische Aufbereitung der Originalmessergebnisse in $\mathbf{0}$ Abb. 1 und 2 .

Interessenkonflikt. Der korrespondierende Autor gibt an, dass kein Interessenkonflikt besteht.

\section{Literatur}

1. Altenmüller E, Diener HC, Dichgans J (1989) Visuell evozierte Potentiale. In: Stöhr M, Dichgans J, Diener HC, Büttner UW (Hrsg) Evozierte Potentiale. 2. Aufl. Springer, Berlin Heidelberg New York, S 279 382

2. Eckhardt-Henn A, Hoffmann SO (Hrsg) (2004) Dissoziative Bewußtseinsstörungen. Schattauer, Stuttgart New York

3. Gast U (2004) Dissoziative Identitätsstörung - valides und dennoch reformbedürftiges Konzept. In Reddemann L, Hofmann A, Gast U (Hrsg) Psychotherapie der dissoziativen Störungen. Georg Thieme Verlag, Stuttgart New York, S 26-36

4. Gast U, Oswald T, Zündorf F (Hrsg) (2000) Das Strukturierte Klinische Interview für DSM-IV Dissoziative Störungen (SKID-D). Deutsche Fassung. Hogrefe, Göttingen

5. Gazzaniga MS (ed) (1999) The New Cognitive Neurosciences. Chpt. V, Attention. Bradford Book, MIT Press, Cambridge MA, pp 619-724

6. Halliday AM (ed) (1982) Evoked potentials in clinical testing. Churchill Livingstone, Edinburgh, p 575

7. Helmholtz H von (1871) Über die Zeit, welche nöthig ist, damit ein Gesichtseindruck zum Bewusstsein kommt. Monatsberichte der Königlichen Preußischen Akademie der Wissenschaften zu Berlin, S 333-337

8. Hillyard SA, Vogel EK, Luck SJ (1998) Sensory gain control (amplification) as a mechanism of selective attention: electrophysiological and neuroimaging evidence. Philosophical Transactions of the Royal Society of London B. Biol Sci 353: 1257-1270

9. Jörg J, Hielscher H (Hrsg) (1993) Evozierte Potentiale in Klinik und Praxis. Eine Einführung in VEP, SEP, AEP, MEP, P300 und PAP. Springer, Berlin Heidelberg New York

10. Kasten E, Wüst S, Behrens-Baumann W, Sabel BA (1998) Computer-based training for the treatment of partial blindness. Nat Med 4: 1083-1087

11. Kastner S (2005) Beyond a relay nucleus: New views on the human LGN. Perception [Suppl] 34

12. Luck SJ, Hillyard SA (1999) The operation of selective attention at multiple stages of processing: Evidence from human and monkey electrophysiology. In: Gazzaniga MS (ed) The New Cognitive Neurosciences. Bradford Book, MIT Press, Cambridge, MA, pp 687-700

13. O'Connor DH, Fukui MM, Pinsk MA, Kastner $S$ (2002) Attention modulates responses in the human lateral geniculate nucleus. Nat Neurosci 5: 1203-1209

14. Pang XD, Bonds AB (1991) Visual evoked potential responses of the anesthetized cat to contrast modulation of grating patterns. Vision Res 31: 15091516

15. Poggel DA (ed) (2002) Effects of visuo-spatial attention on the restitution of visual field defects in patients with cerebral lesions. Shaker-Verlag, Aachen

16. Reddemann L, Hofmann A, Gast U (Hrsg) (2004) Psychotherapie der dissoziativen Störungen. Georg Thieme Verlag, Stuttgart New York

17. Reinders AATS, Nijenhuis ERS, Paans AMJ et al. (2003) One brain, two selves. Neurolmage 20: 2119-2125

18. Reinders AATS, Nijenhuis ERS, Quak J et al. (2006) Psychobiological characteristics of dissociative identity disorder. A symptom provocation study. Biol Psychiatry 60: 730-740

19. Spiegel DS, Cotcomb S, Ren C, Pribram K (1985) Hypnotic hallucination alters evoked potentials. J Abnor Psychol 3: 249-255 
20. Steinberg M (ed) (1994) The structured clinical interview for DSM-IV-dissociative disorders - revised (SCID-D). Am Psychiatric Press, Washington, DC

21. Steinberg M, Hall P, Craig L, Cichitti D (2004) Diagnostik valider und vorgetäuschter Dissoziation mit dem strukturellen klinischen Interview für dissoziative Störungen SCID-D-R - Richtlinien für klinische und forensische Untersuchungen. In: Reddemann L, Hofmann A, Gast U (Hrsg) Psychotherapie der dissoziativen Störungen. Georg Thieme Verlag, Stuttgart New York, S 151-167

22. Strasburger H (Hrsg) (2003) Indirektes Sehen. Formerkennung im zentralen und peripheren $\mathrm{Ge}$ sichtsfeld. Hogrefe, Göttingen Bern Toronto Seattle

23. Strasburger H, Murray IJ, Remky A (1993) Sustained and transient mechanisms in the steadystate visual evoked potential. Onset presentation compared to pattern reversal. Clin Vision Sci 8: 211-234

24. Strasburger H, Remky A, Murray IJ et al. (1996) Objective measurement of contrast sensitivity and visual acuity with the steady-state VEP. Ger J Ophthalmol 5: 42-52

25. Vuilleumier P, Chicherio C, Assal F et al. (2001) Functional neuroanatomical correlates of hysterical sensorimotor loss. Brain 124: 1065-1066

26. Wunderlich K, Schneider KA, Kastner S (2005) Neural correlates of binocular rivalry in the human $L G N$ and V1: An fMRI study. Perception [Suppl] 34

\section{Mittels Datenbank zur passenden Patientenschulung}

Patientenschulung gehört heute zum Standard in der Behandlung und Rehabilitation chronisch Kranker. Doch nicht alle Angebote entsprechen dem aktuellen Erkenntnisstand und gute Programme sind noch nicht genug verbreitet. Die „Datenbank Patientenschulung" wurde von einem Forscherteam der Universität Würzburg als Service für Praktiker entwickelt, die für ihre Patienten ohne aufwändige Recherchen ein Schulungsprogramm suchen.

Derzeit sind 60 Trainingsprogramme in der Datenbank zu finden. Den Grundstock des Internetangebots hat das Würzburger Team aus Literaturrecherchen und Anfragen bei Reha-Einrichtungen und medizinischen Fachgesellschaften im Rahmen des Forschungsprojekts "Zentrum Patientenschulung" gewonnen, einem „Umsetzungsprojekt" der Rehabilitationsforschung. Der traditionelle Arztvortrag sollte um Schulungskonzepte erweitert werden, die eine Aktivierung und Mitwirkung der Patienten fördern. Da sich interaktive Konzepte als besonders wirksam erwiesen haben, gelten Methoden wie praktische Übungen, Kleingruppenarbeit und Rollenspiele als wichtiges Qualitätsund Auswahlkriterium. Aus Expertensicht zeichnen sich gute Schulungen zusätzlich dadurch aus, dass die Patientengruppe geschlossen ist, Lernerfolge überprüft und Nachsorgeangebote und Angehörige mit berücksichtigt werden. Auch sollten Programme von multiprofessionellen Teams stammen. Grundvoraussetzung ist jedoch, dass ein ausgearbeitetes Schulungsmanual vorliegt und das Programm mindestens zwei Sitzungen umfasst.

Die Datenbank stellt die Schulungen mit einer Kurzbeschreibung vor und gibt in einem Raster einen Überblick über Zielgruppen, Themen, Methoden und die didaktischen Ziele, aufgeschlüsselt nach Kategorien wie Wissenserwerb, Fertigkeitentraining, Motivation zur Lebensstiländerung, Angstreduktion und Stärken der sozialen Kompetenz. Sie enthält auch Hinweise auf die nötigen Rahmenbedingungen, Kosten und Bezugsquellen, Fortbildungsangebote und Evaluationsergebnisse. Anhand von Suchfunktionen lassen sich die Programme nach einzelnen Merkmalen filtern, zum Beispiel nach Indikationen: Das sind derzeit Adipositas, Allergien und Asthma, Bluthochdruck und koronare Herzkrankheit, Diabetes, Epilepsie, Hauterkrankungen, Neubildungen, Parkinsonkrankheit, rheumatische Erkrankungen, Pankreatitis, Leber- und Darmerkrankungen, Neurodermitis und chronischer Rückenschmerz. Die Datenbank ist frei zugänglich und soll kontinuierlich erweitert werden.

Dass die Praxis den hohen Qualitätsanforderungen teilweise hinterherhinkt, verdeutlichte eine bundesweite Bestandsaufnahme. Die Rückläufe von 424 Rehabilitationseinrichtungen ergaben, dass von den beschriebenen Schulungen jede dritte auf eine Einzelveranstaltung beschränkt war. Methodisch dominierten Vortrag und Diskussion, nur jede zweite Schulung konnten Patienten in Kleingruppen und mit mehrfach abwechselnden Vermittlungsformen erleben. Eine Ausnahme bildet der Indikationsbereich Rheuma, in dem nach Angaben der Einrichtungen dreiviertel der Schulungen die Qualitätsstandards eines multimodalen, interaktiven Angebots erfüllen und auch manualisiert sind. In der Dermatologie, Allergologie und Pneumologie sind die Verhältnisse ähnlich, jedoch sind seltener Manuale vorhanden, in der Endokrinologie ist es umgekehrt.

www.zentrum-patientenschulung.de/datenbank

Quelle: L. von Manteuffel; 16. Rehabilitationswissenschaftliches Kolloquium, DRV Bund 